\title{
New Scientific Facilities and Directions in Molecular Astrophysics
}

\author{
Arjun Kumar Gautam \\ Department of Physics, Mahendra Multiple Campus, Nepalgunj \\ arjungautamnpj@gmail.com
}

\begin{abstract}
A brief overview is given of major current and future observational facilities which will enable new scientific opportunities in the study of interstellar and circumstellar molecules, ranging from the early universe to protoplanetary disks and exoplanatory atmospheres. Close interaction between astronomers and laboratory astrophysicists will be essential to read the full scientific harvest from these major investments.
\end{abstract}

Keywords: radio telescope, ultra violet telescope, $\mathrm{x}$-ray telescope, optical and near IR telescope, mid and far IR telescope, submillimeter telescope.

\section{INTRODUCTION}

Astronomy and laboratory physics have a long history together, starting centuries ago with the identification of optical lines and features in the solar spectrum (e.g., $\mathrm{He}, \mathrm{H}^{-}$) and in the nebula like Orion (atomic and ionic lines). More recently, observations have expanded to other wavelengths across the electromagnetic spectrum, and have revealed a wealthy of molecular lines at millimeter wavelengths, the identification of which has been possible through laboratory experiments (e.g., identifying 'Xogen' with $\mathrm{HCO}^{+}$). In addition to basic spectroscopy, laboratory and theoretical data have been essential to provide collisional rate coefficients and radiative probabilities necessary to translate observed line fluxes into column densities and abundances, and to derive temperatures and densities from line ratios. The subsequent modeling and interpretation of these abundances requires reaction rates for a wide suite of processes, ranging from photoionisation and photo dissociation crosssections to recombination, ion-molecule, neutralneutral and grain-surface reaction rate coefficients.

Atoms and molecules are found in a wide variety of environments, ranging from the hot and cold diffuse phases of the interstellar medium, to molecular clouds star-forming regions, protoplanetary disks, envelops of evolved stars, stellar atmospheres, cometary coma, and the atmospheres of planets, including those of the recently dis-covered exo-planets. One of the main challenges for the future is to convince the next generation of astronomers that basic atomic and molecular data continue to be essential for the success of their astronomical projects; that existing data bases are still highly incomplete; and that constant maintenance and improvement of them is warranted as new experimental methods and computational facilities are becoming available. Indeed, it is ironic that in this 'golden age' of astronomy, with several billion $\$$ /Euro/Yen facilities (about to come) on line, the investments in laboratory astrophysics have continued to decrease world-wide. Moreover, physics and chemistry university departments have moved in different directions and astronomy can no longer count on significant 'free' efforts from these communities. People with the required skills and expertise are disappearing and there is a general lack of training of young scientists in this interdisciplinary field.

In this paper, a brief overview is given of new observational facilities, which will make exciting new science possible in the coming decades. The summary starts at the shortest wavelengths and moves across the spectrum to long wavelengths. The reader will be encouraged to visit the web sites of the telescopes for more information; these can readily found with any web search engine. Only major facilities are highlighted here. 


\section{OVER VIEW OF NEW FACILITIES}

\subsection{X-ray telescope}

The NASA Chandra and ESA Newton-XMM satellites, launched in the late 1990's, continue to be the workhorse for spectroscopy ranging from soft to hard X-rays. In addition to probing the physics of hot, highly ionized regions such as supernova remnants, they provide constraints on the dust elemental composition (e.g., $\mathrm{Si} / \mathrm{Mg}, \mathrm{Mg} / \mathrm{Fe}$ ratios) and total hydrogen column of molecular gas through absorption measurements, and are thus relevant for molecular astrophysics. The next generation of X-ray telescopes with collecting areas up to $5 \mathrm{~m}^{2}$ and improved spectral resolution are being planned but are unlikely to be launched before 2020 .

\subsection{Radio telescope}

Large aperture radio telescopes operating at $\mathrm{cm}$ wavelengths such as the Effelsberg $100 \mathrm{~m}$ single dish and Very Large Array (VLA) have traditionally focused on $\mathrm{H}$ I $21 \mathrm{~cm}$ observations of the diffuse ISM, with only few molecules detected, mostly $\mathrm{OH}$ and $\mathrm{NH}_{3}$ through their $18 \mathrm{~cm}$ and $1 \mathrm{~cm}$ lines and $\mathrm{H}_{2} \mathrm{O}$ masers at $22 \mathrm{GHz}$. The Greenbank 100m telescope is equipped with receivers up to $50 \mathrm{GHz}$ and has already proven to be very powerful for the detection of the largest complex organic molecules whose low-lying rotational transitions occur at $\mathrm{cm}$ wavelengths. This research would be enhanced by the proposed Square Kilometer Array (SKA), if it has capabilities extending up to $\mathrm{cm}$ wavelengths.

\subsection{Ultraviolet telescopes}

The Copernicus satellite in the early 1980's has opened up the field of UV spectroscopy, which is particularly powerful for probing diffuse translucent clouds. In addition to a wealth of atomic lines, Copernicus observed the Lyman and Werner electronic transitions of $\mathrm{H}_{2}$ and $\mathrm{HD}$ in clouds with visual extinctions along the line of sight of order 0.3 mag (corresponding to a total $\mathrm{H}$ column of a few $\times 10^{-}$ ${ }^{20} \mathrm{~cm}^{2}$ ). Few other molecules have been detected, but UV datd such as that from the International Ultraviolet Explorer also provide constraints on the dust population through the shape of the UV extinction curve and the $2200 \breve{A}$ feature. The Far Ultraviolet Space Explorer (FUSE)has extended this science to fainter, more heavily extincted sources, including transient clouds and studies of the interstellar and intergalactic medium toward nearby galaxies. The next step forward in terms of sensitivity is the Cosmic Origins Spectrograph (COS) with a resolving power $\mathrm{R}=\lambda / \Delta \lambda \leq 24000$, to be installed on the Hubble Space Telescope. The next mission being planned is the World Space Observatory $-\mathrm{UV}$, designed to provide capabilities down to $1020 \breve{A}$.

\subsection{Optical and near-IR telescope}

The majority of the world's telescopes operates at visible and near infrared (IR) wavelengths, from roughly $3000 \breve{A}$ to $2.5 \mu \mathrm{m}$. There are two major developments regarding optical telescopes. The first is a class of new, rapid response/robotic telescopes, designed to (automatically) slew to an area of the sky to observe a recent phenomenon triggered by observations at another wavelength. The best known example is that of gamma-ray bursts. For a few hours after discovery at gamma wavelengths, these sources are very bright at optical wavelengths and excellent targets for high-resolution spectroscopy. Thus, it is possible to obtain spectra of the ISM in galaxies at redshirts of 2 more with the same sensitivity as that toward local $\mathrm{O}$ and $\mathrm{B}$ stars.

The second development is that of the Extremely Large Telescopes (ELTs) with diameter of $\approx 20$ $40 \mathrm{~m}$ being planned across the globe. One of the many technical challenges is to design adaptive optics which can work over large fields, necessary to reach diffraction-limited resolution in the milliarcses range. Because of the high costs, the initial suite of ELT instruments, expected to become operational around 2017, will be limited, and it is not a priori clear when there will be high spectral resolution instruments.

\subsection{Mid-and far-infrared telescopes}

The wavelength range from $2.5-300 \mu \mathrm{m}$ is heavily obscured from the ground and has been opened 
up spectroscopically by the Kuiper Airborne Observatory (KAO) in the 1970's. The Infrared Space Observatory (ISO), which flew from 19951998 , covered the $2.5-200 \mu \mathrm{m}$ range at moderate spectral resolution $(\mathrm{R}=\lambda / \Delta \lambda \leq 3000)$ and was particularly powerful in revealing the wealth of solid state and gaseous features at this wavelength, including the vibrational bands of ices, silicates, oxides, carbides and carbonates; of Polycyclic Aromatic Hydrocarbons (PAHs); and of gas phase molecules including symmetric species like $\mathrm{CH}_{4}, \mathrm{C}_{2} \mathrm{H}_{2}$ and $\mathrm{CO}_{2}$ which cannot be observed at millimeter wavelengths. The pure rotational lines of $\mathrm{H}_{2}$ also occur in this region, as do a number of fine structure lines which are diagnostic of ionized gas and PDRs. The Spitzer Space Telescope, launched in 2003, is currently providing order of magnitude more sensitive spectra than ISO, but over a more limited wavelength range, 5-40 $\mu \mathrm{m}$, and with a resolving power only $\mathrm{R} \approx 100-600$. The Japanese Akari satellite is covering the important $3-5 \mu \mathrm{m}$ range containing strong $\mathrm{H}_{2} \mathrm{O}$ and $\mathrm{CO}_{2}$ ice bands at equally low resolving power. Ground based optical telescopes provide much higher resolution spectroscopy (up to $\mathrm{R} \approx 10^{5}$ ) FROM 3-20Mm, but only in atmospheric windows.

The most powerful mid-IR facility in the future will be the James Webb Space Telescope (JWST), a $6 \mathrm{~m}$-class telescope which is the successor of Hubble and is scheduled for launch around 2013. It will have spectroscopy at $\mathrm{R} \approx 1000-3000$ in the $1-28 \mu \mathrm{m}$ range, with a sensitivity 1-3 orders of magnitude better than ground or Spitzer, allowing spectra of very faint objects ranging from the highest redshift galaxies to the atmospheres of earth like planets. Similarly, mid-IR instruments on ground based ELTs can open up more sensitive high spectral and spatial resolution spectroscopy.

Unique opportunities in this wavelength range will be provided by the Stratosphoric observatory for Infrared Astronomy (SOFIA), A $2.5 \mathrm{~m}$ telescope located in a Boeing 747 airplane which will fly in the stratosphere at $\geq 14 \mathrm{~km}$ altitude, i.e., above $99 \%$ of the earth's water vapor. Equipped with a powerful suite of instruments, it will allow observations of many features at high spectral resolution which are blocked from the ground, albeit with limited sensitivity compared with space missions.

Ground based infrared spectrometers such as the Keck Interferometers and Very Large Telescope Interferometer (VLTI) can achieve unprecedented spatial resolutions down to milli-arcsec, but generally have very limited spectral capabilities and sensitivity. Space based Infrared interferometers such as the ESADARWIN and the NASA Terrestrial Planet Finder (TPF) missions, specially designed to image Earth like planets and search for bio-signatures in their atmospheres, have been proposed but are unlikely to materialize within the next 20 years.

\subsection{Submillimeter telescopes}

It is a 10-meter-wide parabolic dish inside a building to protect it from bad weather. The building front doors and roof are opened when the telescope is in use. The telescope's construction was finished in 1993. Along with the 12 Meter Telescope on Kitt Peak, this telescope is maintained by the Arizona Radio Observatory, a division of Steward Observatory at the University of Arizona. The $3.5 \mathrm{~m}$ Herschel Space Observatory, to be launched in 2008 , will operate from $\approx 60-500 \mu \mathrm{m}$ and will be the most powerful far infrared and submillimeter telescope at wavelengths obscured from Earth. The Heterodyne Instrument for the Far Infrared (HIFI) will have 7 bands with $\mathrm{R} \approx 10^{7}$ up to 1.9 $\mathrm{THz}(160 \mu \mathrm{m})$, where as the Photoconductor Aray Cmer and Spectroeter (PACS)will have a 5x5 pixel spectral imaging covering $55-200 \mu \mathrm{m}$ but at lower spectral resolution $\mathrm{R} \approx 1500$. Herschel will be the only facility for decades that can observe thermal water lines originating from a large range of energy levels, allowing water and related species $(\mathrm{OH}, \mathrm{OI}$, $\mathrm{H}_{3} \mathrm{O}^{+}, \mathrm{HDO}$ ) to be studied in both cold and warm gas. In addition, it will be particularly powerful for complete spectral line surveys unhindered by the atmosphere, and it can probe the lowest rotational transitions of many light hydrides (the building blocks in the gas phase chemical networks). Angular resolution range from $\approx 10^{\prime \prime}$ to $40^{\prime \prime}$. 
The biggest gain in sensitivity and spatial resolution will come from the Atacama Large Millimeter / Submillimeter array (ALMA), a joint NorthAmerica, Europe and East Asia project to be located at the $5000 \mathrm{~m}$ Chajnantor plateau Chile. It will consist of 66 telescopes $(50 \times 12 \mathrm{~m}$ main array, $12 \times 7 \mathrm{~m}$ compact array; $4 \times 12 \mathrm{~m}$ total power dishes), whose signals will be combined through a powerful correlator. At least 6 receiver bands will cover the $\approx 80-900 \mathrm{GHz}$ range. The array will be re-configurable with base lines from $20 \mathrm{~m}$ up to $14 \mathrm{~km}$, providing zoom-lens capabilities with spatial resolutions from a few arcsec down to 0.01 ". This corresponds to linear scales down to a few $\mathrm{AU}$ in the nearest star forming regions, $85 \mathrm{AU}$ at the Galactic center, and $\leq 1 \mathrm{pc}$ in nearby galaxies, ensuring major steps forward in virtually every area of astronomy.

\subsection{Other opportunities}

Progress in theoretical chemistry and molecular dynamics benefits enormously from the much increased computer power over the last years. For example, most of the collisional cross sections used in astrochemistry are provided by quantum chemical calculations of the potential surfaces and subsequent collision dynamics, which become very computer time consuming at high energies.

Laboratory experiments can benefit from major physics facilities, such as synchrotron light sources, (free- electron) laser laboratories, and accelerator facilities.

\section{CONCLUSIONS}

Astronomy is fortunate that various countries and agencies have made major financial investments which will turn our scientific visions and dreams about future facilities into a reality. Many of the new telescopes that will come on line in the next decade are extremely well suited for studying molecular astrophysics. At the same time, this puts the responsibility on the community to make the best use of these telescopes. It is clear that new science areas are opening up rapidly, ranging from the study of molecules in young galaxies with ages only $5 \%$ of the age of the universe to imaging the inner planet -forming zones of disks, searching for even more complex prebiotic molecules, and determining the chemical composition of the atmospheres of exoplanets. A continuing dialogue between observers and laboratory astrophysicists will be key to ensure that these new facilities reach their full scientific potential.

\section{REFERENCES}

[1] Asimov, I. (1979). Isaac Asimov's Book of facts; Sky Watchers. New York: Grosset \& Dunlap. Page 390 - 399. ISBN 0-80389347-7

[2] Hoff HA (Aug 1983). Exosat - the new extrasolar $x$-ray observatory. $J$ Brit Interplan Soc (Space Chronicle). 36 (8): 363-7.

[3] IKI RAN, International Astrophysical Observatory GRANAT Retrieved 2007-1205.

[4] Mandrou P, Jourdain E. et al. (1993). Overview of two-year observations with SIGMA on board GRANAT. Astron Astrophys Supplement Series (97).

[5] Revnivtsev MG, Sunyaev RA, Gilfanov MR, Churazov EM, Goldwurm A, Paul J, Mandrou P, Roques JP (2004). A hard X-ray sky survey with the SIGMA telescope of the GRANAT observatory. Astron Lett. 30 (8): 527-33.

[6] Rohlfs, K., \& Wilson, T. L. (2004). Tools of radio astronomy. Astronomy and astrophysics library. Berlin: Springer.

[7] Sancar J Fredsti, Astronomytoday.com Radio Astronomy

[8] Snow,T.P.,\& McCall, B.J. 2006, ARA \& A $, 44,367$

[9] Van Dishoeck, E.F.,\& Tielens,A.G.G.M. 2001, in The Century of Space Science, ed.J. Bleeker et al.(Dordrecht : Kluwer),607

[10] Van Dishoeck,E.F. 2004, ARA\&A, 42,119 